\title{
Chiral Selection by Interfacial Shearing of Self-Assembled Achiral Molecules
}

\author{
Núria Petit-Garrido, Jordi Ignés-Mullol, Josep Claret, and Francesc Sagués \\ SOC\&SAM group, IN ${ }^{2} U B$ and Departament de Química Física, Universitat de Barcelona, \\ Martí i Franquès 1, 08028 Barcelona, Catalonia, Spain
}

(Received 24 July 2009; published 30 November 2009)

\begin{abstract}
We report a novel phenomenon of chiral selection in self-assembled condensates of achiral amphiphiles. The handedness of chiral textures, reproducing the collective rotational component of the molecular orientation inside submillimeter circular domains, is correlated with the sign of a vortical stirring in the aqueous subphase. We propose an explanation based on the distinctive kinetics of topological defect annihilation during domain coalescence at the initial coarsening stage of a phaseseparating monolayer.
\end{abstract}

PACS numbers: 81.16.Fg, 61.30.Jf, 61.72.Cc, 68.18.-g

Chiral symmetry breaking, i.e., the absence of mirror symmetry, is a pervasive phenomenon encompassing from self-organizing galaxies to the molecular architecture of biopolymers $[1,2]$. In materials science this is a relevant issue in self-assembling processes [3], as well as in asymmetric catalysis [4]. Although spontaneous chiral resolution has been observed during aggregation of either chiral or achiral molecules [5], we are in general unable to realize a robust process of chiral selection. In particular, it is totally unexplored whether the latter may occur at the level of a single layer of molecules under a bulk chiral force, such as a circularly polarized light or a vortical flow. Here we show that the molecular orientational chirality in labile condensates of an achiral surfactant may be selected by stirring. Segregated submillimeter circular domains at the air-water interface display, when observed by optical reflection microscopy, a preferred sign of their tangentially assembled molecules (chiral textures) that correlates with that of an underneath vortical flow. This selection process results from the shear-assisted coalescence of elliptic nuclei of achirally packed molecules. Different from dichroism-based techniques [6,7] applied to supramolecular aggregates $[8,9]$, we here directly observe how a macroscopic chiral force may be imprinted downscale during bottom-up molecular assembling.

The experimental system is a monomolecular layer (Langmuir monolayer) of an azobenzene-based insoluble amphiphile at the air-water interface. Its relevant thermodynamic parameters are the temperature $T$ and the surface pressure $\Pi$, defined as the reduction of the interfacial tension with respect to that of the bare interface. The presence of the azobenzene moiety makes this surfactant photosensitive, featuring two isomeric forms (cis or folded, and trans or elongated) that may be interconverted by light of suitable wavelength [10]. Lateral orientational coherence in Langmuir monolayers can be registered as Brewster-angle-microscopy (BAM) textures $[11,12]$ that reflect the spatial variations of the local optical axis of the material [13]. Earlier BAM observations in our chosen system have revealed that monolayers of the cis isomer are isotropic and featureless, while monolayers of the trans isomer form birefringent mesophases. The light-induced evolution of a monolayer initially of the cis form leads to a two-dimensional biphasic emulsion composed of circular trans-enriched domains, embedded in a nearly pure cis matrix [14]. The organization of trans molecules inside the confined domains is characterized by a uniform tilt with respect to the air-water interface normal so molecular ordering can be described by a two-dimensional vector field. Constant angle anchoring at the domain boundary [14] results in the inclusion of inner point defects of total charge $s=+1[15,16]$. Although the amphiphilic compound is achiral, each circularly evolved trans-rich domain displays a random chirality, here defined as the collective rotational component of the $X Y$ vector field (orientational chirality). In absence of subphase stirring, domains with clockwise $(\mathrm{CW})$ and counter clockwise $(\mathrm{CCW})$ orientations are equally represented. This spontaneous symmetry breaking is reminiscent of the two-dimensional chiral resolution previously reported in solid supported (LangmuirBlodgett) films $[17,18]$, but totally different from the enantiomorphic discrimination observed sometimes in Langmuir monolayers prepared from chiral surfactants [19], a topic extensively reviewed in the literature [20].

In our experiments, we have developed a protocol that results in the selection of circular trans-rich domains of the desired chirality. We pour approximately $40 \mathrm{ml}$ of pure water (Milipore MiliQ quality) in a Petri dish (8 cm diameter, $2.5 \mathrm{~cm}$ height $)$ and stir it at a constant rate $\Omega(0<$ $\Omega<1600 \mathrm{rpm}$ ) by means of a reversible magnetic stirrer (J. P. Selecta, Spain) with a submerged cylindrical Tefloncoated magnetic bar (8 $\mathrm{mm}$ long, $3 \mathrm{~mm}$ diameter). Monolayers are prepared by depositing at the outer part of the vortical funnel one drop $(2 \mu \mathrm{l})$ of the spreading chloroform (Baker p.a.) solution (approximately $1 \mathrm{mM}$ ) of the azobenzene amphiphile 4-[4-[(4-octylphenyl/azo]phenoxy]butanoic acid, that was custom synthesized [21]. This solution is previously irradiated with UV light for 
10 min to ensure maximum presence of the cis isomer. Experiments are conducted at room temperature $\left(24.0-26.0^{\circ} \mathrm{C}\right)$ and the resulting surface pressure lays in the range $0-1 \mathrm{mN} \mathrm{m}^{-1}$. Stirring is applied for 5 min under white light illumination (halogen lamp with IR cutoff filter, $30 \mathrm{~mW} \mathrm{~cm}^{-2}$ ) to enhance cis-trans conversion. After stopping both stirring and illumination, BAM images of the monolayer are recorded during approximately $30 \mathrm{~min}$. Recording during stirring is unpractical, due to curvature of the interface and the low sensitivity of high-speed cameras.

We quantify the extent of chiral selection in terms of the enantiomeric excess of $\mathrm{CW}$ domains. We denote such an excess $e e_{\mathrm{CW}}$ and it is counted as $\left(2 n_{\mathrm{CW}}-n_{T}\right) / n_{T} \times 100$. Here, $n_{\mathrm{CW}}$ stands for the number of $\mathrm{CW}$ domains with respect to the total number $n_{T}$. In a typical experiment, $n_{T}$ ranges between 700 and 800 . The sign of the rotation defines two branches, where a different chirality is selected for the majority of the two-dimensional domains. Experimental results indicate that a neat handedness selection takes place and correlates with the sign of the spinning magnetic bar: $\mathrm{CW}$ (resp. CCW) stirring favors domains with a collective $\mathrm{CW}$ (resp. CCW) bendlike distortion. Total discrimination is never observed, even for the most extreme shearing conditions investigated $(1600 \mathrm{rpm})$. Instead, enantiomeric excesses increase monotonously up to a saturation value slightly above $40 \%$ for roughly $1000 \mathrm{rpm}$. Surface particle-image-velocimetry (PIV) measurements confirm that surface flow speeds increase steadily with the stirring rate within the entire range of considered values. Results of our experiments are summarized in Fig. 1 and in [22].

Given the impossibility to directly observe the monolayer under stirring, we choose to monitor the twodimensional phase separation for the interface at rest. BAM resolution limits our ability to resolve the shape and texture of condensed trans domains to those larger than about $20 \mu \mathrm{m}$. At this stage domains are elliptic and display a rather uniform reflectivity. An analysis of the inner molecular field suggests that field lines are symmetrical with respect to the long diameter of the domain, departing from a point defect on one of the poles. Field lines converge towards the opposed pole, although their sink is a virtual defect: an imaginary point located outside of the domain (Fig. 2 and [22]). At this point we cannot provide a microscopic explanation either for the anisotropic shape of the elliptic domains or for the asymmetry of the molecular field. Nevertheless, we previously reported the tendency of field lines to be parallel to the domain boundary. It can be shown that this condition, along with the elliptic domain shape results in the formation of point singularities of charge $s=+1 / 2$ at both poles. Moreover, asymmetry between head and tail in Langmuir monolayers is compatible with the observed asymmetry of the molecular field.

Our observations also point towards the existence of long-range attractions between domains, presumably of

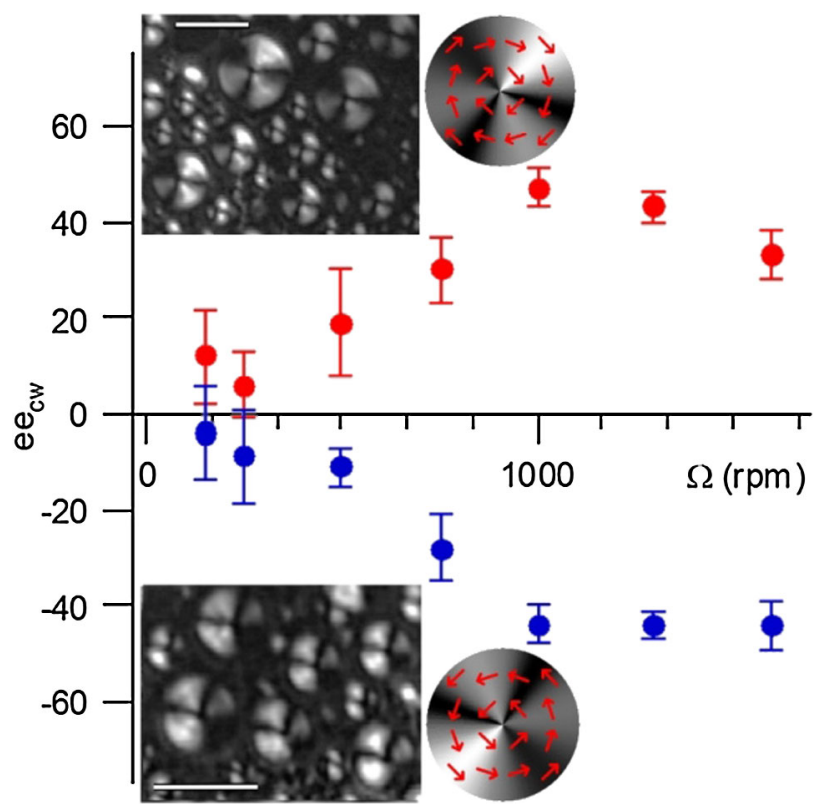

FIG. 1 (color online). The enantiomeric excess, $e e_{\mathrm{CW}}$, is plotted as a function of the rotating rate of the stirrer, $\Omega$. BAM images with an ensemble of the chiral mesostructures after 5 minutes under 1000 r.p.m. stirring are shown next to each branch, along with a sketch of the resolved in-plane orientational field. Qualitatively, BAM textures appear as three black brushes, with either two upwards brushes (CCW orientation) or two downwards brushes (CW orientation). The maximum reflectivity appears in the first and third quadrants of the domains for $\mathrm{CW}$ and $\mathrm{CCW}$ orientation, respectively. White bar corresponds to $100 \mu \mathrm{m}$.

electrostatic origin. This favors the lateral coalescence of drifting elliptic nuclei to give rise to larger aggregates whose textures are different depending on the relative orientational fields of the parent domains. We observe that the fusion of condensates with parallel molecular orientation leads, in a simple process, to larger elliptical domains that keep the common configuration. Conversely, if domains with antiparallel orientations merge, the shape of the resulting condensate becomes circular and the chiral bendlike texture appears (Fig. 2 and [22]). In addition, elliptical domains may merge with already formed circular domains leading to a larger circular domain with the same chirality as its parent.

In the following, we will focus on the second scenario reported above, which leads to the formation of a chiral entity from the merging of two achiral units on a quiescent subphase. Actually, the handedness of the resulting circular domain depends on the individual molecular orientations of the parent couple: a configuration of eastward- (resp. westward-) north-westward- (resp. eastward-) south domains gives rise to a $\mathrm{CW}$ (resp. $\mathrm{CCW}$ ) texture. The remarkable change in both the shape and collective orientational field accompanying this coalescence process is a complex, defect-mediated, phenomenon, illustrated with an example in Fig. 2 and sketched in Fig. 3, corre- 
a
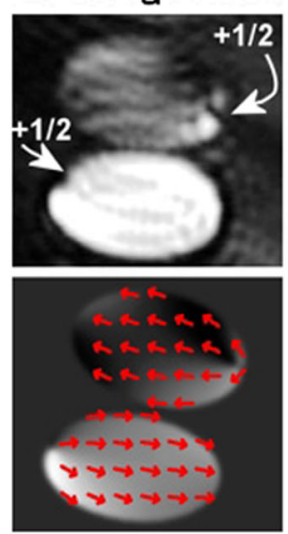

b
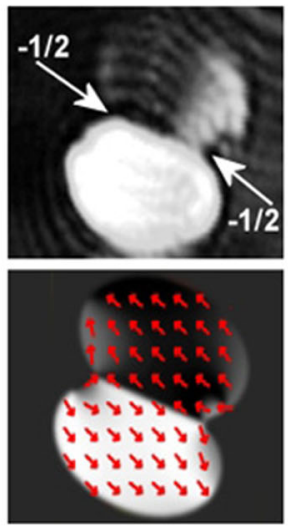

c
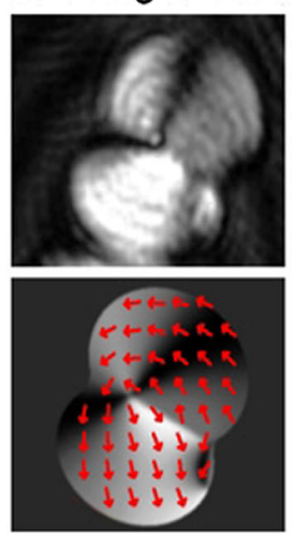

d
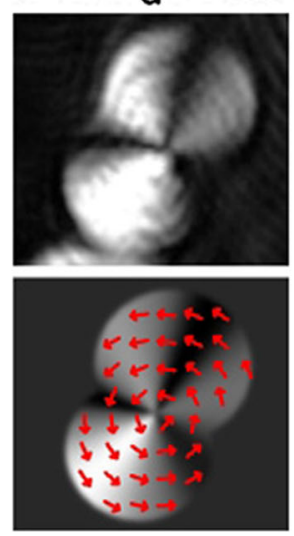

e
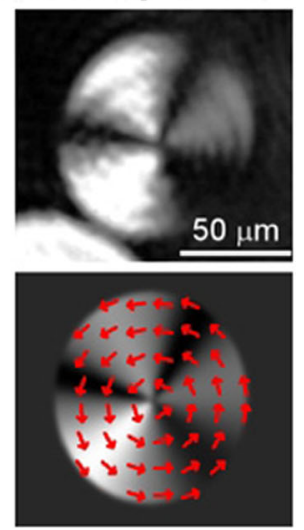

FIG. 2 (color online). Sequence of BAM images showing the fusion of two antiparallel elliptical domains (a) that leads to the formation of a circular domain with $\mathrm{CCW}$ orientational order (e). A sketch of the in-plane orientational field along with the corresponding simulated BAM image is shown below each snapshot. Elapsed time between frames (b) and (d) is $74 \mathrm{~s}$ (see movie of sequence and high resolution render of frame (b) in [22]). Line tension promotes relaxation into a circular shape (e).

sponding to the formation of a CCW chiral domain. First a pair of elliptic domains must approach each other and collide with an appropriately aligned configuration [panels 2(a) and 3(a)]. When they laterally contact, a pair of $s=$ $-1 / 2$ defects form at the intersection points [panels 2 (b) and 3(b)]. Each of the newly created defects approaches and annihilates with the respectively closest $s=+1 / 2$ real defect located at the poles of the parent domains. Subsequently, the remaining virtual $(s=+1 / 2)$ defects move towards the fused domain from their respective escaped poles [panel 3(c)] and enter into the domain [panels 2(c) and 3(d)], pulled by a disclination line. The latter finally relaxes [panels 2(d) and 3(e)] by shrinking until collapse, leading to the single $s=+1$ defect, central

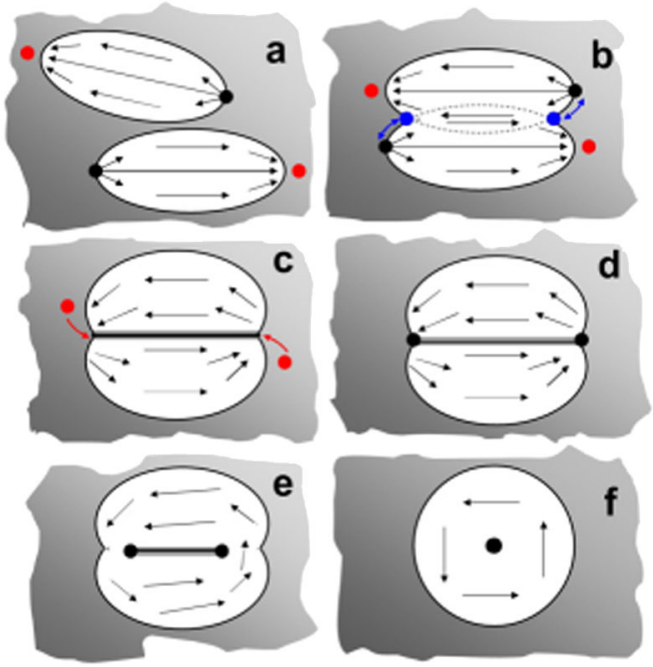

FIG. 3 (color online). Sketch of the point defect-mediated mechanism leading to the formation of a chiral circular domain with CCW orientation from the coalescence of two antiparallel elliptical domains. Real $+1 / 2$ (black), intersection $-1 / 2$ [blue (dark gray)] and virtual $+1 / 2$ [red (medium gray)] defects are depicted. to the resulting domain, whose outer boundary progressively rounds up to a circular contour (panels 2(e) and 3(f) and [22]).

In order to understand the chiral selection process under stirring, we need to reexamine the just proposed fusion mechanism when the monolayer is sheared by the subphase vortical flow created by the spinning magnet. Notice that a doublet of elliptic domains with parallel but opposed molecular orientations is indeed a chiral unit, with the eastwards-north/westwards-south configuration being enantiomorphic of the westwards-north/eastwards-south ensemble. It is thus not surprising that both entities may behave differently facing a vortical flow. In fact, the latter imposes a shear to the monolayer whose rate is maximal at the center of the funnel and decays outwards (it is reported to decay radially as $r^{-2}$ while the linear velocity decreases radially as $r^{-1}$ from the center of the funnel [23]). Contact between domains is thus favored by their nonzero relative drifting velocity, with the inner domain, more central to the vortex, always catching its outer, more eccentric, mate. In the case of, say, a CW flow [Fig. 4(a)], the landing of the westwards-inner domain on the eastwards-outer one will

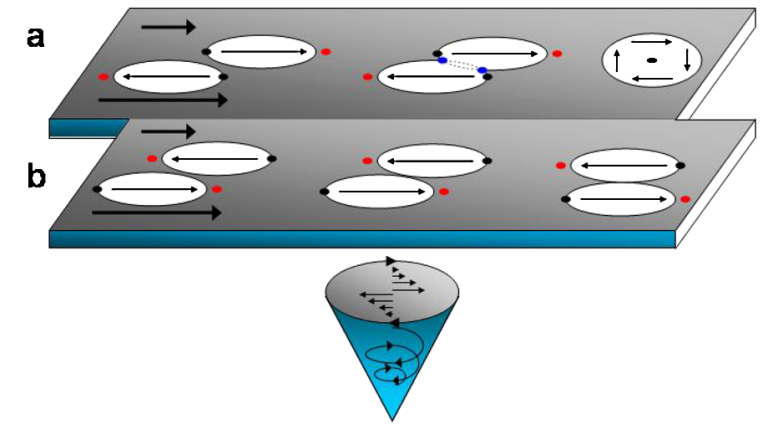

FIG. 4 (color online). Proposed mechanism for chiral symmetry selection in the presence of a subphase $\mathrm{CW}$ vortical flow. Two different situations arise depending on the relative position of the two antiparallel elliptical domains, as described in the text. 
place the $s=-1 / 2$ intersection defects close to the real $s=+1 / 2$ located at the domains poles. In this way, their mutual pairwise rapid annihilation is sterically enhanced and kinetically favored. Conversely, the enantiomorphic configuration [Fig. 4(b)] with the eastwards-inner domain being drifted to collapse into its westwards-outer mate, is sterically hindered and kinetically unfavored, since the $s=$ $-1 / 2$ defects are closer now to virtual rather than to real pole-based $s=+1 / 2$ defects. We argue, consistently with the observation of the monolayer at rest as depicted in Fig. 2, that annihilation of virtual defects, although not topologically forbidden, is much slower than for real ones. Notice that our argument is finally based upon kinetic considerations for a defect-assisted coalescence process. This scenario may also account for the observed lack of an absolute chiral selection, regardless of the stirring rate applied to the interfacial system, since both processes depicted in Fig. 4 are possible, but the one leading to the circular domains of the chosen chirality is faster.

Some remarks are worth making as a concluding paragraph. Our observations demonstrate a robust mechanism of chiral selection in sheared monolayers of condensed surfactants that self-assemble in labile mesophases. This process is particularly striking since chiral selection takes place at a different spatial dimension and at a disparate length scale with respect to those of the force that imprints it: a genuinely three-dimensional, centimeter-scale, chiral bulk force is transferred into two-dimensional, tens of micron-size, interfacial self-assemblies. Finally we recall at this point recent observations [24] on discrimination of chiral solid states, by employing glass beads to continuously grind the microcrystallites formed during stirring of aqueous dissolutions of either inorganic [25] or organic chiral materials [26]. These grinding experiments lead, in clear contrast to ours, to a chiral predominance whose ultimate sign is unpredictable. Our results unveil a new perspective into the classical problem of the origin of homochiral terrestrial life [27,28], based on the potential to elicit a particular handedness in biopolymers from templates [29] moulded by interfacial flows of primordial achiral components. We also anticipate possibilities for enantioselective catalysis, either directly on aqueous interfaces [30], or by further transfer of the monolayer to a solid support [31].

We acknowledge financial support by MICINN (Project No. FIS2006-03525) and by DURSI (Project No. 2005 SGR 00653). N.P. acknowledges support from MICINN (Grant No. AP2007-01103).

[1] L. D. Barron, Chirality in Natural and Applied Science (Blackwell, Oxford, 2002), p. 53.

[2] P. Cintas, Angew. Chem., Int. Ed. 41, 1139 (2002).
[3] G. M. Whitesides, J. P. Mathias, and C. Seto, Science 254, 1312 (1991).

[4] I. Lee, Z. Ma, S. Kaneko, and F. Zaera, J. Am. Chem. Soc. 130, 14597 (2008).

[5] L. Pérez-Garcia and D. Amabilino, Chem. Soc. Rev. 36, 941 (2007).

[6] A. Tsuda, M. A. Alam, T. Harada, T. Yamaguchi, N. Ishii, and T. Aida, Angew. Chem., Int. Ed. 46, 8198 (2007).

[7] G. P. Spada, Angew. Chem., Int. Ed. 47, 636 (2008).

[8] J. M. Ribó, J. Crusats, F. Sagués, J. Claret, and R. Rubires, Science 292, 2063 (2001).

[9] O. Ohno, Y. Kaizu, and H. Kobayashi, J. Chem. Phys. 99, 4128 (1993).

[10] H. Rau, Photochemistry and Photophysics (CRC Press, Boca Raton, Florida, 1990), p. 119.

[11] S. Hénon and J. Meunier, Rev. Sci. Instrum. 62, 936 (1991).

[12] D. Hönig and D. Möbius, J. Phys. Chem. 95, 4590 (1991).

[13] T. M. Fischer, R. F. Bruinsma, and C. M. Knobler, Phys. Rev. E 50, 413 (1994).

[14] J. Ignés-Mullol, J. Claret, R. Reigada, and F. Sagués, Phys. Rep. 448, 163 (2007).

[15] The topological charge of a point defect is defined as the number of turns performed by the orientational field along a close circuit surrounding the singularity, and it will be negative if the two senses of rotation are opposite.

[16] N. D. Mermin, Rev. Mod. Phys. 51, 591 (1979).

[17] R. Viswanathan, J. A. Zasadzinski, and D. K. Schwartz, Nature (London) 368, 440 (1994).

[18] P.Z. Guo, L. Zhang, and M. H. Liu, Adv. Mater. 18, 177 (2006).

[19] D. Andelman and P. G. de Gennes, C.R. Acad. Sci. Paris, Ser. 2 307, 233 (1988).

[20] N. Nandi and D. Vollhardt, Chem. Rev. 103, 4033 (2003).

[21] J. Crusats, R. Albalat, J. Claret, Ignés-Mullol, and F. Sagués, Langmuir 20, 8668 (2004).

[22] See EPAPS document No. E-PRLTAO-103-064949 for additional images and movie files. For more information on EPAPS, see http://www.aip.org/pubservs/epaps.html.

[23] G. Hálasz, B. Gyure, I. M. Janosi, K. G. Szabo, and T. Tél, Am. J. Phys. 75, 1092 (2007).

[24] J. M. McBride and J. Tully, Nature (London) 452, 161 (2008).

[25] C. Viedma, Phys. Rev. Lett. 94, 065504 (2005).

[26] W. L. Noorduin, T. Izumi, A. Millemaggi, M. Leeman, H. Meekes, W. J. P. V. Enckevort, R. M. Kellogg, B. Kaptein, E. Vlieg, and D. G. Blackmond, J. Am. Chem. Soc. 130, 1158 (2008).

[27] L. D. Barron, Space Sci. Rev. 135, 187 (2008).

[28] R. Plasson, D. K. Kondepudi, H. Bersini, A. Commeyras, and K. Asakura, Chirality 19, 589 (2007).

[29] R. M. Hazen, Am. Mineral. 91, 1715 (2006).

[30] H. Zepik, E. Shavit, M. Tang, T. R. Jensen, K. Kjaer, G. Bolbach, L. Leiserowitz, I. Weissbuch, and M. Lahav, Science 295, 1266 (2002).

[31] K. Katsonis, H. Xu, R. M. Haak, T. Kudernac, Z. Tomovicacute, S. George, M. V. der Auweraer, A. P. H. J. Schenning, E.W. Meijer, and B.L. Feringa, Angew. Chem., Int. Ed. 47, 4997 (2008). 